\title{
Anomaly induced chiral magnetic current in a Weyl semimetal: Chiral electronics
}

\author{
Dmitri E. Kharzeev ${ }^{1,2, *}$ and Ho-Ung Yee ${ }^{3, \dagger}$ \\ ${ }^{1}$ Department of Physics and Astronomy, Stony Brook University, Stony Brook, New York 11794-3800, USA \\ ${ }^{2}$ Department of Physics, Brookhaven National Laboratory, Upton, New York 11973-5000, USA \\ ${ }^{3}$ Department of Physics, University of Illinois, Chicago, Illinois 60607, USA \\ and RIKEN-BNL Research Center, Brookhaven National Laboratory, Upton, New York 11973-5000, USA
}

(Received 16 July 2012; published 11 September 2013)

\begin{abstract}
Electric circuits involving Weyl semimetals possess unusual properties induced by the quantum anomaly. The chiral magnetic current in a Weyl semimetal subjected to magnetic field modifies the behavior of such circuits in a drastic way. We consider two explicit examples: (i) a circuit involving the "chiral battery" and (ii) a circuit that can be used as a "quantum amplifier" of magnetic field. The unique properties of these circuits stem from the chiral anomaly and may be utilized for creating "chiral electronic" devices.
\end{abstract}

DOI: 10.1103/PhysRevB.88.115119

PACS number(s): 72.20.My, 72.25.Dc, 75.85.+t

Recently, the 3D materials with linearly dispersing excitations ${ }^{1}$ have attracted significant attention. The existence of these "chiral" excitations stems from the point touchings of conduction and valence bands. The corresponding dynamics is described by the Hamiltonian $H= \pm v_{F} \boldsymbol{\sigma} \cdot \boldsymbol{k}$, where $v_{F}$ is the Fermi velocity of the quasiparticle, $\boldsymbol{k}$ is the momentum in the first Brillouin zone, and $\sigma$ are the Pauli matrices. This Hamiltonian describes massless particles with positive or negative (depending on the sign) chiralities, e.g., neutrinos, and the corresponding wave equation is known as the Weyl equation, hence the name Weyl semimetal. ${ }^{1}$ Weyl semimetals are closely related to $2 \mathrm{D}$ graphene, ${ }^{2}$ and to the topological insulators ${ }^{3}$ 3D materials with a gapped bulk and a surface supporting chiral excitations. Specific realizations of Weyl semimetals have been proposed, including a multilayer structure composed of identical thin films of a magnetically doped 3D topological insulator, separated by ordinary-insulator spacer layers. ${ }^{4}$

Weyl semimetals provide a unique opportunity to study the macroscopic behavior of systems composed by chiral fermions. In particular, they allow ${ }^{5}$ to study, in a condensed matter system, the chiral magnetic effect expected, ${ }^{6-10}$ and possibly observed experimentally at Relativistic Heavy Ion Collider, ${ }^{11}$ in chirally imbalanced quark-gluon plasma in the presence of an external magnetic effect as a consequence of axial anomaly in $\mathrm{QCD} \times \mathrm{QED}$. Closely related phenomena have been discussed in the physics of neutrinos, ${ }^{12}$ conductors with mirror isomer symmetry, ${ }^{13,14}$ primordial electroweak plasma, ${ }^{15}$ and quantum wires. ${ }^{16}$ Note that the role of axial anomaly and the corresponding Chern-Simons dynamics are crucial for the existence of the chiral magnetic current; without the anomaly, this current has to vanish in thermal equilibrium, in contrast to naive arguments. The effects of the anomaly on the transport in Weyl semimetals, including the chiral magnetic effect, have recently been investigated in. ${ }^{17-20}$

In this paper, we would like to consider some of the electric circuits involving Weyl semimetals. We will argue that the existence of chiral magnetic current in Weyl semimetal subjected to magnetic field can cause an interesting, and potentially useful for practical applications, behavior of such circuits. To be specific, we will consider two explicit examples: (i) a circuit involving the chiral battery; ${ }^{9}$ and (ii) a circuit that can be used as an amplifier of magnetic field, possibly opening a way to creating a sensor of ultraweak magnetic fields.
Consider first a cylindrical sample of Weyl semimetal put inside a solenoid that provides an external, constant magnetic field of strength $B$ along the longitudinal direction of cylindrical geometry, say $\hat{x}^{3}$, see Fig. 1 . The top and bottom of the Weyl semimetal are touching metallic plates that can conduct electric currents flowing through the sample. These two metallic plates are then connected to an outside circuit which is characterized by a resistance $R$. Let the cross section area of the Weyl semimetal sample be $A$ and the longitudinal length be $d$.

When an external magnetic field is present, there exists an anomaly-induced chiral magnetic current density along $\hat{x}^{3}$, given by

$$
J_{a}=\frac{e^{2}}{4 \pi^{2}} \sum_{i} k_{i} \mu_{i} B
$$

where $\mu_{i}$ is the chiral chemical potential specifying the difference between the chemical potential of excitations with opposite chiralities, and the sum is over different kinds of band touching Weyl points ("flavors" of chiral fermions). The chiral chemical potential can either be a property of the Weyl semimetal, ${ }^{5}$ or can be stored in the system through the anomaly equation in parallel electric and magnetic fields. ${ }^{9}$ However in the former case it is a conserved quantity (for a fixed geometry of the sample) and thus does not give rise to the chiral magnetic current in equilibrium. Once an external magnetic field is applied, the energy stored in the difference of the chemical potentials of left- and right-handed fermions can be released by generating the current [Eq. (1)], hence the name chiral battery. ${ }^{9}$

Note that according to Eq. (1) a Weyl semimetal is a kind of battery that provides a definite amount of current, contrary to conventional batteries that support a definite voltage. The total anomaly-induced current through the sample is $I_{a}=A J_{a}$, where $A$ is the area. However, this is not the entire current. Let the entire current be $I$, then there is a voltage drop along the resistance $R$ given by $\Delta V=I R$. Since the same amount of voltage drop should also occur along the Weyl semimetal sample, there is an electric field along $\hat{x}^{3}$ direction with a magnitude

$$
E=-\frac{\Delta V}{d}=-\frac{I R}{d} ;
$$




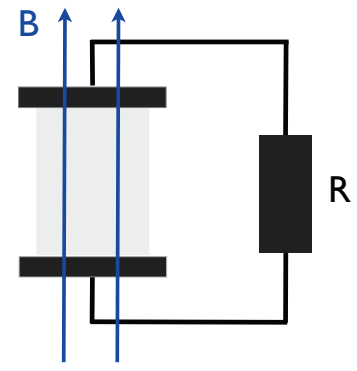

FIG. 1. (Color online) The chiral battery: Weyl semimetal (shown in gray) connected to the circuit with resistance $R$ in an external magnetic field $B$.

note the negative sign of $E$. This electric field gives rise to a normal current through conductivity $\sigma$

$$
I_{n}=A \sigma E=-\frac{A \sigma R}{d} I=-\frac{R}{R_{0}} I,
$$

where $R_{0} \equiv \frac{d}{\sigma A}$ is the intrinsic resistance of the Weyl semimetal sample. The total current $I$ should be the sum of $I_{a}$ [Eq. (1)] and $I_{n}$ [Eq. (3)], that is determined self-consistently as

$$
\begin{aligned}
I & =I_{a}+I_{n}=A \frac{e^{2}}{4 \pi^{2}} \sum_{i} k_{i} \mu_{i} B-\frac{R}{R_{0}} I \\
& =\frac{A}{1+\frac{R}{R_{0}}} \frac{e^{2}}{4 \pi^{2}} \sum_{i} k_{i} \mu_{i} B=\frac{I_{a}}{1+\frac{R}{R_{0}}} .
\end{aligned}
$$

This is the equation governing the performance of the chiral battery.

Let us now see how the energy discharge works for the chiral battery. From the total current [Eq. (4)] through the resistance $R$ and the normal current $I_{n}$ through $R_{0}$, the energy discharge rate can be found using Eqs. (3) and (4) as

$$
\frac{d \mathcal{E}}{d t}=R I^{2}+R_{0} I_{n}^{2}=\frac{R}{\left(1+\frac{R}{R_{0}}\right)} A^{2}\left(\frac{e^{2}}{4 \pi^{2}} \sum_{i} k_{i} \mu_{i}\right)^{2} B^{2} .
$$

This should match the reduction of internal energy of the Weyl semimetal sample. In the presence of both electric field $E$ as in Eq. (2) and the magnetic field $B$, the charge density of the $i$ th Weyl point changes via triangle anomaly as

$$
\frac{d \rho_{i}}{d t}=\frac{k_{i} e^{2}}{4 \pi^{2}} \boldsymbol{E} \cdot \boldsymbol{B}=-\frac{k_{i} e^{2}}{4 \pi^{2}} \frac{I R}{d} B .
$$

The total volume of the sample is $A d$, so that the total rate of increase of the $i$ th charge is

$$
\frac{d Q_{i}}{d t}=-\frac{k_{i} e^{2}}{4 \pi^{2}} A I R B
$$

from which the rate of internal energy change is

$$
\begin{aligned}
\frac{d \mathcal{E}_{\text {int }}}{d t} & =\sum_{i} \mu_{i} \frac{d Q_{i}}{d t}=-\sum_{i} \frac{k_{i} \mu_{i} e^{2}}{4 \pi^{2}} A I R B \\
& =-\frac{R}{1+\frac{R}{R_{0}}} A^{2}\left(\frac{e^{2}}{4 \pi^{2}} \sum_{i} k_{i} \mu_{i}\right)^{2} B^{2},
\end{aligned}
$$

using the expression [Eq. (4)] for $I$, which indeed agrees precisely with Eq. (5). The time dependence of the chiral

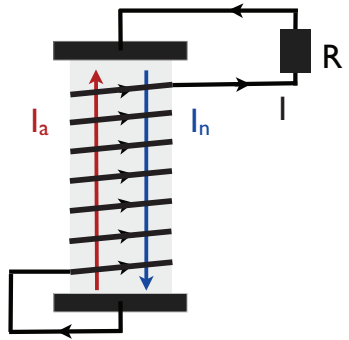

FIG. 2. (Color online) The quantum amplifier: Weyl semimetal (shown in gray) wrapped by a solenoid that is connected to the circuit with resistance $R$. The chiral magnetic anomalous current $I_{a}$ is generated in response to magnetic field; the resulting voltage drop across the Weyl semimetal sample induces a normal component of the current $I_{n}$. The total current flowing through the resistance is $I=I_{a}+I_{n}-d Q / d t$, where $Q$ is the charge accumulated in the capacitor formed by the metal leads attached to the sample (shown in black); note that $I_{n}$ is negative, see Eq. (12).

battery performance relies on the detailed equation of state relating $\rho_{i}$ and $\mu_{i}$.

Although the above example requires an external magnetic field, one can think of another setup that is completely self-driven, as shown in Fig. 2. A cylindrical Weyl semimetal sample is put between two metallic plates that form a capacitor with capacitance $C$. The longitudinal distance and the transverse area of the sample are again $d$ and $A$. In addition there is a solenoid that wraps the Weyl semimetal sample $N$ times over the distance $d$; this solenoid is connected to the two metal plates. An external circuit with a resistance $R$ is loaded to the solenoid circuit. Let the total current along the solenoid and the external resistance $R$ be $I$. The strength of the induced magnetic field along $\hat{x}^{3}$ inside the solenoid is

$$
B=\frac{N}{d} I,
$$

from the Maxwell's equation $\nabla \times \boldsymbol{B}=\boldsymbol{J}$, which gives rise to the anomaly-induced current:

$$
I_{a}=A J_{a}=A \frac{e^{2}}{4 \pi^{2}} \sum_{i} k_{i} \mu_{i} B=\left(\frac{e^{2}}{4 \pi^{2}} \sum_{i} k_{i} \mu_{i}\right) \frac{A N}{d} I .
$$

Let the charge stored inside the capacitor formed by the two metallic plates be $Q$. Since the voltage drop along the capacitor is $\Delta V=Q / C$, there exists a longitudinal electric field (note the negative sign from our definition of $Q$ )

$$
E=-\frac{\Delta V}{d}=-\frac{1}{C d} Q,
$$

and we have a normal current along the Weyl semimetal sample:

$$
I_{n}=A \sigma E=-\frac{A \sigma}{C d} Q=-\frac{1}{C R_{0}} Q,
$$

where $R_{0}$ is the intrinsic resistance of the sample. The charge conservation law for the capacitor dictates

$$
\frac{d Q}{d t}=I_{a}+I_{n}+I,
$$


whereas the Kirchhoff's law of vanishing voltage drop along a closed circuit trajectory gives us

$$
I R+L \frac{d I}{d t}+\frac{1}{C} Q=0,
$$

where $L \equiv \frac{A N^{2}}{d}$ is the inductance of the solenoid. Using Eqs. (10) and (12), the equations (13) and (14) acquire the form

$$
\begin{gathered}
\frac{d Q}{d t}=\left[\left(\frac{e^{2}}{4 \pi^{2}} \sum_{i} k_{i} \mu_{i}\right) \frac{A N}{d}+1\right] I-\frac{1}{R_{0} C} Q, \\
\frac{d I}{d t}=-\frac{R}{L} I-\frac{1}{L C} Q .
\end{gathered}
$$

These equations comprise a complete dynamical system given an initial data $\left(I_{0}, Q_{0}\right)$ at $t=0$, and assuming that the chemical potentials $\mu_{i}$ are approximately constant during the discharge process. The time evolution of this system can be solved analytically by the Ansatz

$$
\left(\begin{array}{c}
Q(t) \\
I(t)
\end{array}\right)=\left(\begin{array}{l}
C_{1} \\
C_{2}
\end{array}\right) e^{\lambda_{ \pm} t},
$$

where the characteristic exponents $\lambda_{ \pm}$of the above system are solutions of

$$
\begin{aligned}
\lambda^{2} & +\left(\frac{1}{R_{0} C}+\frac{R}{L}\right) \lambda+\frac{R}{R_{0} L C} \\
& +\frac{1}{L C}\left[\left(\frac{e^{2}}{4 \pi^{2}} \sum_{i} k_{i} \mu_{i}\right) \frac{A N}{d}+1\right]=0,
\end{aligned}
$$

one of which has a positive real part, and hence instability, if

$$
-\left(\frac{e^{2}}{4 \pi^{2}} \sum_{i} k_{i} \mu_{i}\right)>\frac{d}{A N}\left(1+\frac{R}{R_{0}}\right) .
$$

When this condition is met, the system has a particular mode in a discharging phase which develops an exponentially increasing current $I$ (and $Q$ ).

To see how it can be used for detecting an ultraweak magnetic field, let us introduce a small background external magnetic field $B_{\text {ext }}$ that is to be detected. The equation (9) is modified to

$$
B=\frac{N}{d} I+B_{\mathrm{ext}},
$$

so that the above equations of motion (15) and (16), now including an external magnetic field $B_{\text {ext }}$, become

$$
\begin{aligned}
\frac{d Q}{d t}= & {\left[\left(\frac{e^{2}}{4 \pi^{2}} \sum_{i} k_{i} \mu_{i}\right) \frac{A N}{d}+1\right] I } \\
- & \frac{1}{R_{0} C} Q+A\left(\frac{e^{2}}{4 \pi^{2}} \sum_{i} k_{i} \mu_{i}\right) B_{\mathrm{ext}}, \\
& \frac{d I}{d t}=-\frac{R}{L} I-\frac{1}{L C} Q,
\end{aligned}
$$

where we have a new source term in the first equation proportional to the external magnetic field. The above inhomogeneous linear differential equation can be solved to give

$$
\left(\begin{array}{c}
Q(t) \\
I(t)
\end{array}\right)=C_{+}\left(\begin{array}{c}
Q_{+} \\
I_{+}
\end{array}\right) e^{\lambda_{+} t}+C_{-}\left(\begin{array}{c}
Q_{-} \\
I_{-}
\end{array}\right) e^{\lambda_{-} t}+\left(\begin{array}{c}
Q_{p} \\
I_{p}
\end{array}\right)
$$

where $\left(Q_{ \pm}, I_{ \pm}\right)^{T}$ are the eigenvectors of the eigenvalue equation with our previous eigenvalues $\lambda_{ \pm}$,

$$
\left[\begin{array}{cc}
-\frac{1}{R_{0} C}\left(\frac{e^{2}}{4 \pi^{2}} \sum_{i} k_{i} \mu_{i}\right) \frac{A N}{d}+1 \\
-\frac{1}{L C} & -\frac{R}{L}
\end{array}\right]\left(\begin{array}{c}
Q_{ \pm} \\
I_{ \pm}
\end{array}\right)=\lambda_{ \pm}\left(\begin{array}{c}
Q_{ \pm} \\
I_{ \pm}
\end{array}\right),
$$

and $\left(Q_{p}, I_{p}\right)^{T}$ is a particular solution sourced by the external magnetic field,

$$
Q_{p}=\frac{\left(\frac{e^{2}}{4 \pi^{2}} \sum_{i} k_{i} \mu_{i}\right) A R C}{\left(\frac{e^{2}}{4 \pi^{2}} \sum_{i} k_{i} \mu_{i}\right) \frac{A N}{d}+1+\frac{R}{R_{0}}} B_{\mathrm{ext}}, \quad I_{p}=-\frac{1}{R C} Q_{p} .
$$

The integration constants $C_{ \pm}$are determined by the initial condition which is naturally $Q(0)=I(0)=0$. For any nonzero $B_{\text {ext }}$, one finds that $C_{ \pm}$are proportional to $B_{\text {ext }}$. In particular $C_{+} \neq 0$ is induced by having $B_{\text {ext }}$, so that the mode growing exponentially as a function of time is indeed triggered by the external magnetic field $B_{\text {ext }}$.

One can control the instability condition (19) by varying the external resistance $R$ of the circuit. Increasing $R$ beyond a critical value

$$
R>R_{c} \equiv R_{0}\left[-\left(\frac{e^{2}}{4 \pi^{2}} \sum_{i} k_{i} \mu_{i}\right) \frac{A N}{d}-1\right]
$$

will remove the instability from the system, so that the device can stay in a stable condition. One can decrease $R$ below $R_{c}$ for a detection of an ultraweak magnetic field.

Let us see whether our instability condition (19) can be met with reasonable parameters of the current Weyl semimetals. We take $5 \mathrm{meV}$ (millielectron volts) as a typical value for the chemical potential of Weyl semimetals, and assume a system of a centimeter size. Note that our formulas are based on the unit system where $\hbar=c=1$, so that the length and energy are related by $1 \mathrm{~cm}^{-1}=2 \times 10^{-2} \mathrm{meV}$, and the fine structure constant is $\frac{e^{2}}{4 \pi} \equiv \alpha=\frac{1}{137}$. With these values, the l.h.s. of Eq. (19) is

$$
1.2 \times 10^{-2}\left(\frac{\sum_{i} k_{i} \mu_{i}}{-5 \mathrm{meV}}\right) \mathrm{meV},
$$

whereas the r.h.s. of Eq. (19) is

$$
1.0 \times 10^{-2}\left(\frac{d}{1 \mathrm{~cm}}\right)\left(\frac{1 \mathrm{~cm}^{2}}{A}\right)\left(\frac{20}{N}\right)\left(\frac{1+\frac{R}{R_{0}}}{10}\right) \mathrm{meV} .
$$

We see that the condition (19) for the instability can easily be met with a centimeter size of Weyl semimetal device.

As the system evolves in time, at some point one can no longer treat $\mu_{i}$ constant and the system goes out of the discharging phase [Eq. (19)], so the current $I$ starts to decrease. This happens because of the triangle anomaly that yields for the $i$ th charge density

$$
\frac{d \rho_{i}}{d t}=\frac{k_{i} e^{2}}{4 \pi^{2}} \boldsymbol{E} \cdot \boldsymbol{B}=-\frac{k_{i} e^{2}}{4 \pi^{2}}\left(\frac{N}{C d^{2}} I Q+\frac{Q}{C d} B_{\mathrm{ext}}\right),
$$


using Eqs. (11) and (20) for electric and magnetic fields. The previous equations (21) and (22), combined with the above equation (29), form a more complete set of dynamical equations governing the time evolution of the system. One needs the equations of state

$$
\mu_{i}=\mu_{i}\left(\left\{\rho_{j}\right\}\right)
$$

to solve the system in detail for a concrete realization of the Weyl semimetal. Around the Weyl points, the dispersion relation of the Weyl excitations is linear in momentum

$$
\epsilon=v|\mathbf{p}|,
$$

with an effective "speed of light" $v$. In the regime of $\mu \gg T$ (low temperature regime), the density from the Fermi-Dirac distribution with the above dispersion relation is given by

$$
\rho \approx \int_{|\mathbf{p}|<p_{F}} \frac{d^{3} p}{(2 \pi)^{3}}=\frac{p_{F}^{3}}{6 \pi^{2}}=\frac{\mu^{3}}{6 \pi^{2} v^{3}},
$$

using $\mu=v p_{F}$. In the opposite regime of high temperature $T \gg \mu$, the same computation gives

$$
\rho \approx \frac{T^{2}}{6 v^{3}} \mu .
$$

The above equations of state (32) and (33) can be used to solve the time evolution of the system after our unstable mode is triggered by an external magnetic field. Since the room temperature $T_{r} \approx 25 \mathrm{meV}$ is larger than $\mu \approx 5 \mathrm{meV}$, the latter relation (33) can be used to describe a device operating at a room temperature.
Let us provide an exemplar solution of the time evolution assuming a centimeter size device operating at the room temperature with velocity $v=0.01$ (in units of $c$ ). We assume the value of an external magnetic field to be $B_{\text {ext }}=$ $10^{-8}$ Gauss $=0.5 \mathrm{~cm}^{-2}$ which is about the magnetic field in the human brain. We also take the resistance $R_{0}=R=1$ which is dimensionless in our unit system. Noting that the capacitance and the inductance are given by

$$
C=\frac{A}{d}=1 \mathrm{~cm}, \quad L=\frac{A N^{2}}{d}=400 \mathrm{~cm},
$$

with $A=1 \mathrm{~cm}^{2}, d=1 \mathrm{~cm}$, and $N=20$, our equations (21), (22), and (29) become

$$
\begin{aligned}
\frac{d Q}{d t} & =(0.046 \mu+1) I-Q+1.2 \times 10^{-3} \mu, \\
\frac{d I}{d t} & =-\frac{1}{400} I-\frac{1}{400} Q, \\
2.6 \times 10^{11} \frac{d \mu}{d t} & =-0.046 I Q-1.2 \times 10^{-3} Q,
\end{aligned}
$$

where different quantities are measured in the following units: The electric charge $Q$ is dimensionless, the current $I$ is measured in $\mathrm{cm}^{-1}$, the chemical potential $\mu$ in $\mathrm{cm}^{-1}$, time in $\mathrm{cm}$.

These units can be easily converted into conventional ones by restoring the speed of light $c=3 \times 10^{10} \mathrm{~cm} / \mathrm{sec}$ and noting that the charge in Coulombs can be obtained from our (dimensionless) charge by dividing by $6.24 \times 10^{18}$. For example, the typical current on Fig. 3 is $I=2 \times 10^{6} \mathrm{~cm}^{-1}=$
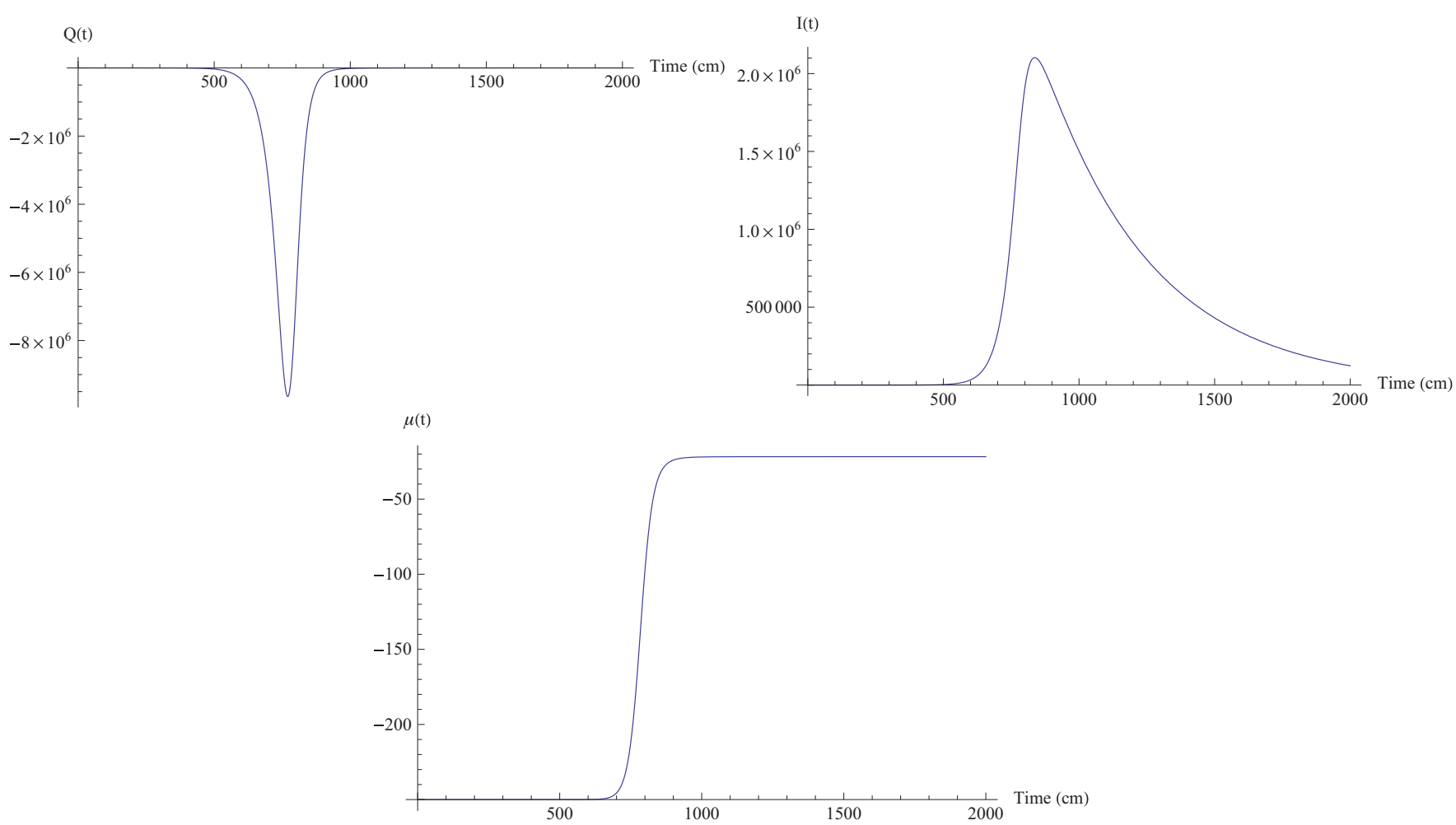

FIG. 3. (Color online) The numerical solution of Eqs. (21), (22), and (29) with the parameters, $B_{\text {ext }}=10^{-8}$ Gauss and $v=0.01$, for a centimeter size device operating at the room temperature. In conventional units, $I=2 \times 10^{6} \mathrm{~cm}^{-1}=0.01$ Amperes, and the typical time scale is $1000 \mathrm{~cm}=30 \mathrm{~ns}$ (nanoseconds). 
0.01 Amperes, and the typical time scale is $1000 \mathrm{~cm}=30 \mathrm{~ns}$ (nanoseconds).

The initial condition is $I(0)=Q(0)=0$ and $\mu(0)=$ $-5 \mathrm{meV}=-2.5 \times 10^{2} \mathrm{~cm}^{-1}$. In Fig. 3, we show the numerical solution of $Q(t), I(t)$, and $\mu(t)$ as a function of time. As we see in the plots, the system initially develops an exponentially large signal triggered by the external magnetic field before the signal eventually dies off. We have checked numerically that this feature is robust and is observed for a wide range of parameters.

Even though we need the equation of state to evaluate the detailed time evolution of the system, there exists a sufficiently general and interesting feature of the circuit of Fig. 2. Namely, the instability of that circuit driven by quantum anomaly makes it a quantum amplifier of magnetic field. In principle, this instability can be induced by a single quantum of magnetic flux through the sample. Therefore the considered circuit may be utilized as a sensor of ultraweak magnetic fields.

The circuits discussed above represent only a couple of examples from a vast array of devices that one can envision. We hope that the chiral electronics based on Weyl semimetal circuits can serve as a fascinating way to explore the macroscopic dynamics induced by the chiral anomaly, and perhaps open a path towards new electronic devices.

We thank G. Başar, G. Dunne, L. Levitov, M. Stephanov, M. Ünsal, and I. Zahed for useful discussions. This research was supported by the US Department of Energy under Contracts DE-AC02-98CH10886 and DE-FG-88ER41723.
*Dmitri.Kharzeev@stonybrook.edu

†hyee@uic.edu

${ }^{1}$ X. Wan, A. M. Turner, A. Vishwanath, and S.Y. Savrasov, Phys. Rev. B 83, 205101 (2011).

${ }^{2}$ A. K. Geim and K. S. Novoselov, Nat. Mater. 6, 183 (2007).

${ }^{3}$ C. L. Kane and E. J. Mele, Phys. Rev. Lett. 95, 146802 (2005); B. A. Bernevig, T. L. Hughes, and S.-C. Zhang, Science 314, 1757 (2006); J. E. Moore and L. Balents, Phys. Rev. B 75, 121306(R) (2007); G. E. Volovik, Lect. Notes Phys. 718, 31 (2007), and references therein.

${ }^{4}$ A. A. Burkov and L. Balents, Phys. Rev. Lett. 107, 127205 (2011).

${ }^{5}$ A. A. Zyuzin, S. Wu, and A. A. Burkov, Phys. Rev. B 85, 165110 (2012).

${ }^{6}$ D. Kharzeev, Phys. Lett. B 633, 260 (2006).

${ }^{7}$ D. Kharzeev and A. Zhitnitsky, Nucl. Phys. A 797, 67 (2007).

${ }^{8}$ D. E. Kharzeev, L. D. McLerran, and H. J. Warringa, Nucl. Phys. A 803, 227 (2008).
${ }^{9}$ K. Fukushima, D. E. Kharzeev, and H. J. Warringa, Phys. Rev. D 78, 074033 (2008).

${ }^{10}$ D. E. Kharzeev, Annals Phys. 325, 205 (2010).

${ }^{11}$ B. I. Abelev et al. (STAR Collaboration), Phys. Rev. Lett. 103, 251601 (2009).

${ }^{12}$ A. Vilenkin, Phys. Rev. D 20, 1807 (1979); 22, 3067 (1980); 22, 3080 (1980).

${ }^{13}$ G. M. Eliashberg, Zh. Eksp. Teor. Fiz. 38, 188 (1983).

${ }^{14}$ L. S. Levitov, Yu.V. Nazarov, and G. M. Eliashberg, Zh. Eksp. Teor. Fiz. 88, 229 (1985).

${ }^{15}$ M. Giovannini and M. E. Shaposhnikov, Phys. Rev. Lett. 80, 22 (1998); Phys. Rev. D 57, 2186 (1998).

${ }^{16}$ A.Y. Alekseev, V.V. Cheianov, and J. Frohlich, Phys. Rev. Lett. 81, 3503 (1998).

${ }^{17}$ D. T. Son and N. Yamamoto, Phys. Rev. Lett. 109, 181602 (2012).

${ }^{18}$ I. Zahed, Phys. Rev. Lett. 109, 091603 (2012).

${ }^{19}$ D. T. Son and B. Z. Spivak, arXiv:1206.1627.

${ }^{20}$ A. A. Zyuzin and A. A. Burkov, Phys. Rev. B 86, 115133 (2012). 